\title{
Comparative assessment of the bacterial communities associated with Aedes aegypti larvae and water from domestic water storage containers
}

Nsa Dada ${ }^{*}$, Estelle Jumas-Bilak ${ }^{2,3}$, Sylvie Manguin ${ }^{4}$, Razak Seidu' ${ }^{1}$, Thor-Axel Stenström ${ }^{1,5}$ and Hans J Overgaard ${ }^{1,6,7}$

\begin{abstract}
Background: Domestic water storage containers constitute major Aedes aegypti breeding sites. We present for the first time a comparative analysis of the bacterial communities associated with Ae. aegypti larvae and water from domestic water containers.

Methods: The $16 \mathrm{~S}$ rRNA-temporal temperature gradient gel electrophoresis (TTGE) was used to identify and compare bacterial communities in fourth-instar Ae. aegypti larvae and water from larvae positive and negative domestic containers in a rural village in northeastern Thailand. Water samples were cultured for enteric bacteria in addition to TTGE. Sequences obtained from TTGE and bacterial cultures were clustered into operational taxonomic units (OTUs) for analyses.

Results: Significantly lower OTU abundance was found in fourth-instar Ae. aegypti larvae compared to mosquito positive water samples. There was no significant difference in OTU abundance between larvae and mosquito negative water samples or between mosquito positive and negative water samples. Larval samples had significantly different OTU diversity compared to mosquito positive and negative water samples, with no significant difference between mosquito positive and negative water samples. The TTGE identified 24 bacterial taxa, belonging to the phyla Proteobacteria, Firmicutes, Actinobacteria, Bacteroidetes and TM7 (candidate phylum). Seven of these taxa were identified in larval samples, 16 in mosquito positive and 13 in mosquito negative water samples. Only two taxa, belonging to the phyla Firmicutes and Actinobacteria, were common to both larvae and water samples. Bacilli was the most abundant bacterial class identified from Ae. aegypti larvae, Gammaproteobacteria from mosquito positive water samples, and Flavobacteria from mosquito negative water samples. Enteric bacteria belonging to the class Gammaproteobacteria were sparsely represented in TTGE, but were isolated from both mosquito positive and negative water samples by selective culture.
\end{abstract}

Conclusions: Few bacteria from water samples were identified in fourth-instar Ae. aegypti larvae, suggesting that established larval bacteria, most likely acquired at earlier stages of development, control the larval microbiota. Further studies at all larval stages are needed to fully understand the dynamics involved. Isolation of enteric bacteria from water samples supports earlier outcomes of E. coli contamination in Ae. aegypti infested domestic containers, suggesting the need to further explore the role of enteric bacteria in Ae. aegypti infestation.

Keywords: Aedes aegypti, Bacterial diversity, Enteric bacteria, E. coli, Domestic water storage containers, $16 \mathrm{~S}$ rRNA-TTGE, Thailand

\footnotetext{
* Correspondence: nsadada@yahoo.com

${ }^{1}$ Department of Mathematical Sciences and Technology, Norwegian

University of Life Sciences, Ås, Norway

Full list of author information is available at the end of the article
}

\section{Biomed Central}

(c) 2014 Dada et al.; licensee BioMed Central Ltd. This is an Open Access article distributed under the terms of the Creative Commons Attribution License (http://creativecommons.org/licenses/by/4.0), which permits unrestricted use, distribution, and reproduction in any medium, provided the original work is properly credited. The Creative Commons Public Domain Dedication waiver (http://creativecommons.org/publicdomain/zero/1.0/) applies to the data made available in this article unless otherwise stated. 


\section{Background}

The container-breeding mosquito, Aedes aegypti, is a wellrecognized vector of diseases of significant public health concern, such as dengue fever, yellow fever, and chikungunya [1]. Aedes aegypti is known to breed mainly in domestic water containers in and around human dwellings [2-4]. These containers are ecosystem microcosms supporting food webs that are dependent on bacteria [5]. Studies conducted on the microbial ecology of Aedes breeding containers have mainly focused on the invasive Ae. albopictus as well as other tree-hole mosquitoes e.g. Ae. triseriatus and Toxorhynchites rutilus. These studies have explored the microbial communities in natural containers such as tree holes, discarded or unused containers (e.g. tyres), and ornamental containers (e.g. plant pots and cemetery urns) [5-8] with no focus on the microbial ecology of Ae. aegypti infested domestic water containers. One study on the effect of Ae. aegypti midgut microbiota on its susceptibility to DENV-2 however characterized the bacterial content of domestic water containers from which the mosquitoes were collected [9].

Mosquito-microbe interactions are of increasing research interests [10]. These interactions which span from pathogenic to obligate symbiosis [11], usually affect the evolutionary success and physiological functions of the mosquitoes. Such functions could be beneficial to the mosquitoes, for example, in the synthesis of essential nutrients that may be lacking from food sources, and conferral of resistance to pathogens [10,12], or detrimental, where the microbiota directly interfere with mosquito development and fitness $[10,13]$. These interactions are being explored for the development of novel control strategies for mosquito vectors and mosquito-borne diseases [12], an approach recently termed 'symbiotic control' [14].

Bacteria constitute a proportion of mosquito microbiota, colonizing a variety of mosquito organs, chiefly the midgut, and to a lesser extent the hemolymph, salivary glands and reproductive organs $[10,11]$. The bacterial communities within mosquitoes vary depending on the mosquito species, sex, stage of development and habitat [10]. The origin of bacteria within mosquitoes and the dynamics involved in bacterial colonization are unclear as both exogenous and endogenous factors are known to affect the initial colonization and nature of the bacterial composition [10]. Some studies show that immature mosquitoes or newly emerged adults harbor bacteria derived from their breeding habitats $[10,15]$. Others show that the bacterial communities in blood feeding adults are influenced by their blood meals [16]. Feeding may, thus, play a role in determining bacterial communities within mosquitoes. The bacteria within mosquitoes have also been shown to be acquired transstadially [17]. Yet it is still uncertain whether these bacteria acquired transstadially or through feeding are able to survive and colonize the mosquitoes, or are transient and lost during digestion and/or molting [10]. A better understanding of the dynamics involved in bacterial colonization within mosquitoes is therefore still needed.

Furthermore, understanding the mosquito-bacteria interactions within Ae. aegypti infested domestic containers would be beneficial for Ae. aegypti control. Thus far, one study that has examined the bacterial content of Ae. aegypti infested domestic containers [9] employed conventional culture-dependent methods. These methods are unable to capture non-cultivable bacteria that may be present within samples, thus leading to an incomplete picture of the bacterial communities. This contributes to the vague knowledge or limited information available on the dynamics involved in the mosquito-bacteria interactions. However, molecular methods such as the temporal temperature gradient gel electrophoresis (TTGE) and 16S rRNA sequencing can identify both cultivable and non-cultivable bacteria although not without limitations [18].

In this study, the 16S rRNA-TTGE technique was used to comparatively assess the bacterial communities associated with Ae. aegypti larvae and water from domestic water containers. We hypothesized that the bacteria in Ae. aegypti infested containers are determinants of Ae. aegypti production and thus may constitute a major proportion of the larval microbiota. This was based on our previous study where significantly more Ae. aegypti pupae were produced in domestic containers contaminated with Escherichia coli compared to containers that were not contaminated with E. coli [19]. To test this hypothesis and update information on the bacterial communities associated with $A e$. aegypti larvae and water in Ae. aegypti infested domestic containers, the abundance and diversity of bacterial taxa between Ae. aegypti larvae and water from Ae. aegypti positive and negative containers were determined and compared.

\section{Methods}

\section{Sampling sites and sample collection}

The study was conducted in February 2012 following our earlier study on the relationship between Ae. aegypti production and $E$. coli contamination in domestic containers in Thailand and Laos [19]. Mosquito and water samples were collected from domestic water containers in Waileum village, Khon Kaen province, Thailand, where 25 out of 122 houses included in the previous study were randomly selected. Out of the randomly selected houses, 17 had at least one mosquito positive container, and were included in this study. The other eight houses were excluded because they were either unoccupied at the time of sampling or had no mosquito positive containers.

In each house, water samples were collected from each mosquito positive container and one mosquito negative container directly into $100 \mathrm{ml}$ standard 
Whirl-Pak ${ }^{\circledast}$ Thio-Bags ${ }^{\oplus}$. Water was vigorously mixed prior to sampling to ensure that biofilms were included. A total of 25 Ae. aegypti positive containers (10 cement tanks, 12 earthen jars, two plastic buckets and one plastic drum), and 17 negative containers (13 earthen jars, two cement tanks, one plastic drum and one metal pot) were sampled. A sample of ten 4th instar larvae (or all if less than ten) was collected from each mosquito positive container and transferred to sterile $15 \mathrm{ml}$ Eppendorf tubes. All water and larval samples were transported on dry ice to the laboratory and stored in $-80^{\circ} \mathrm{C}$ until further processing.

\section{Sample preparation for DNA extraction}

Samples were thawed at room temperature before DNA extraction. Aedes aegypti larvae were identified using illustrated keys [20], and all Ae. aegypti larvae (mean $4 \pm$ 1) per container were pooled together for analysis. A total of 25 pools of Ae. aegypti larvae from 25 mosquito positive containers were analyzed. Larval samples were surface sterilized; first rinsed in 70\% ethanol, then suspended in $70 \%$ ethanol and agitated with a vortex mixer for about 10 seconds, and finally rinsed with sterile DNA free water.

Each water sample was filtered through a cellulose nitrate membrane filter $(0.45 \mu \mathrm{m}$ pore size, $47 \mathrm{~mm}$ dia., Sartorius Stedium ${ }^{\odot}$ ) using aseptic vacuum filter units (Millipore ${ }^{\oplus}$ ). $0.45 \mu \mathrm{m}$ pore size membrane filters were used due to high turbidity of some water samples. Each membrane filter was cut in two halves; one half cut into small pieces and placed in sterile $1.5 \mathrm{ml}$ Eppendorf tubes for DNA extraction and the other placed on Trypticase soy agar (TSA) plates for bacterial cultures and further isolation of enteric bacteria. Prior to water sample filtration, sterile DNA free water was filtered through each filter unit (using separate membranes) as negative controls to check for contamination.

\section{Isolation of enteric bacteria from water samples}

One half of each filter membrane placed on TSA plates were incubated at $37 \pm 0.5^{\circ} \mathrm{C}$ for 48 hours. Colonies from positive TSA plates were cryopreserved in Tryptic soy broth (TSB) with $15 \%$ glycerol at $-80^{\circ} \mathrm{C}$ for isolation of enteric bacteria. Enteric bacteria were isolated from preserved samples by streaking on Drigalski agar (DA) plates. Following incubation at $37 \pm 0.5^{\circ} \mathrm{C}$ for 48 hours, each colony displaying distinct morphotypes on positive DA plates were isolated and sub-cultured on fresh DA plates to obtain pure single colonies. Pure single colonies were cryopreserved in TSB with $15 \%$ glycerol at $-80^{\circ} \mathrm{C}$ for subsequent DNA extraction.

\section{Genomic DNA extraction}

Bacterial genomic DNA was extracted from pools of sterilized Ae. aegypti larvae and bacterial cells retained on the other half of each filter membrane using the Master Pure Gram Positive DNA purification kit ${ }^{\circ}$ (Epicentre Biotechnologies, Madison, USA) following manufacturer's instructions. Each sample was disrupted in $150 \mu \mathrm{l}$ Tris-EDTA (TE) buffer solution with the aid of sterile plastic pestles before DNA extraction.

DNA from pure bacterial colonies was extracted by boiling. Cryopreserved pure bacterial colonies were regrown on TSA at $37 \pm 0.5^{\circ} \mathrm{C}$ for 24 hours prior to DNA extraction. Three to six colonies, depending on size, were picked from TSA plates and mixed with $100 \mu \mathrm{l}$ of DNA free water in sterile $1.7 \mathrm{ml}$ Eppendorf tubes. The cell suspensions were held in a boiling water-bath for $10 \mathrm{~min}$ to lyse the cells, then vigorously homogenized with a vortex mixer for $10 \mathrm{sec}$ and chilled on ice. Resulting DNA samples were stored in $-20^{\circ} \mathrm{C}$ for PCR.

\section{PCR amplification of bacterial small subunit rRNA gene and TTGE analysis}

Bacterial DNA from mosquito larvae and water samples were used as PCR templates. For mosquito and uncultured water samples, the V2-V3 region of the $16 \mathrm{~S}$ rRNA gene was amplified using universal $16 \mathrm{~S}$ rRNA bacterial primers HDA1: 5'-ACTC CTA CGG GAG GCA GCA GT-3', and HDA2: 5'-GTATTA CCG CGG CTG CTG GCA-3', which yield PCR fragments of $\sim 199 \mathrm{bp}$. The $16 \mathrm{~S}$ rRNA gene from cultured water samples was amplified using the primers 27f: 5'-GTGCTGCAGAGAGTTTG ATCCTGGCTCAG-3'), and 1492r: 5'-CACGGATCCT ACGGGTACCTTGTTACGACTT-3', which yield PCR fragments of $1469 \mathrm{bp}$. Amplification was carried out following previously reported protocols [21] and [18] respectively. PCR products obtained from mosquito larvae and uncultured water samples were separated by TTGE. TTGE migration was performed following a previously reported protocol [21] on $16 \mathrm{~cm} \times 16 \mathrm{~cm} \times 1 \mathrm{~mm}$ gels using the DCode universal mutation detection system (Bio-Rad Laboratories, Marne-la-Coquette, France). PCR products from pure bacterial cultures and about $50 \%$ of the bands produced by TTGE were sequenced. The remaining bands were assigned to an Operational Taxonomic Unit (OTU) by comparing their migration distance to that of sequenced bands.

\section{Sequence analysis, taxonomy assignment and alignment}

The sequences were analyzed using the Quantitative Insights Into Microbial Ecology (QIIME) software package version 1.7.0 [22]. Bacterial diversity of a sample analyzed using TTGE is generally reflected by the number of bands on the TTGE profile, each band usually representing an OTU. However, Manguin et al. [21] reported bands with different migration distances belonging to the same OTU due to sequence heterogeneity among their $16 \mathrm{~S}$ rRNA gene copies. This could lead to an overestimation of 
the bacterial diversity. Therefore to avoid this, sequences obtained from TTGE as well as those obtained from water sample cultures were clustered into OTUs, prior to analysis. Sequences were clustered into OTUs using the UCLUST pipeline [23] in QIIME at an identity threshold of $97 \%$ (i.e. sequences that were $97 \%$ similar were binned into the same OTU). The most abundant sequence within each cluster was selected as a representative of the OTU. The resulting OTUs were assigned to taxa using the Ribosomal Database Project (RDP-II) classifier [24] trained on Greengenes reference database [25] via QIIME at a minimum confidence score of $80 \%$. The OTUs were aligned against the Greengenes core reference alignment [26] using the Python Nearest Alignment Space Termination (PyNAST) alignment algorithm [27] in QIIME with a minimum identity of $75 \%$. Relative abundance of bacterial taxa was computed and collated using the make_OTU_table.py and summarize_taxa. py scripts in QIIME. This was visualized on histograms and compared using two-sample t-tests in Excel. A phylogenetic tree, required to run diversity analyses down the QIIME pipeline, was constructed using the FastTree approximately maximum likelihood program [28] in QIIME. Representative OTU sequences generated from this study are available in the GenBank database [GenBank:KJ814977 - KJ815004; KM108474 - KM108577].

\section{Diversity analysis}

Diversity analysis was performed separately on bacterial sequences obtained by TTGE (mosquito and uncultured water samples), and on those obtained from water samples cultures. The OTU diversity within and between samples were compared, using alpha $(\alpha)$ and beta $(\beta)$ diversity indices respectively. Alpha diversity was measured with the Shannon-Wiener index (relative OTU abundance and evenness), Observed species metrics (OTU abundance), and Faith's whole tree Phylogenetic Diversity (branch length-based diversity) [29]. Alpha diversity means and two-sample t-tests were computed using Excel. Beta diversity was evaluated using the unweighted UniFrac [30] pipeline in QIIME. Principal Coordinate Analysis ( $\mathrm{PCoA}$ ) was used to interpret and visualize the variation in UniFrac distance matrix. The largest amount of variation is explained by the first principal coordinate (PCo1), the second largest by the second principal coordinates (PCo2) and so on. To test the strength and significance of the PCoA, the adonis function in PRIMER 6 (PRIMER-E Ltd., Luton, UK) was used on unweighted UniFrac distance matrices via QIIME. The adonis function computes an effect size value $R^{2}$, which shows the percentage of variation in distance matrices explained by the sample. Two-sample student's t-test to compare mean unweighted UniFrac distances between samples was calculated using the dissimilarity_mtx_stats.py script in QIIME with results presented in boxplots. Prior to diversity analyses samples were rarefied down to the mean number of sequences per sample - four for samples analyzed by TTGE, and two for water sample cultures - in ten iterations to standardize number of sequence per sample. Level of statistical significance for all analyses was set at $\mathrm{p} \leq 0.05$.

\section{Results}

\section{Data summary}

TTGE profiles were obtained from 18 (72\%) pooled mosquito samples, 22 (88\%) mosquito positive and 16 (80\%) mosquito negative water samples. The remaining samples showed no PCR amplification or faint PCR signals leading to undetectable TTGE profiles. A total of 236 sequences were generated from these TTGE bands; 61 from mosquito larvae, 117 and 58 from mosquito positive and negative water samples respectively. The sequences were binned into 134 unique OTUs, with 1-14 sequences per OTU, and average sequence length of $120 \mathrm{bp}$. Seven of the unique OTUs failed to align to any bacterial small subunit models (SSU) and were discarded. Sequences from 12 pools of mosquitos, 11 mosquito positive containers, and 10 mosquito negative containers were included in diversity analyses after rarefaction.

Water samples from all mosquito negative containers (17) and 23 (92\%) mosquito positive containers produced bacterial colonies on selective media for enteric bacteria. A total of 111 bacterial sequences were obtained from these colonies; 69 from mosquito positive containers and 42 from mosquito negative containers. These sequences were binned into 24 unique OTUs, with 1-5 sequences per OTU, and average sequence length of $911 \mathrm{bp}$. Seven of the OTUs failed to align to any bacterial SSU models and were discarded. Sequences from 19 mosquito positive, and 13 mosquito negative water samples were included in diversity analyses after rarefaction.

\section{Alpha diversity of bacterial OTUs}

Figure 1 shows alpha diversity rarefaction curves based on the Shannon Wiener index, observed species index and Faith's phylogenetic diversity for sequences generated by TTGE. Mosquito positive water samples had the highest OTU abundance, while mosquito larvae had the lowest across all the three indices (Figure 1). Two-sample t-tests comparing each index between samples revealed statistically significant results for only the Shannon-Wiener index, which was significantly higher in mosquito positive water samples compared to mosquito larvae $(\mathrm{p}<0.05)$. The difference was not statistically significant between mosquito larvae and mosquito negative water samples, or between mosquito positive and negative water samples.

For sequences obtained from bacterial cultures of water samples, OTU abundance was higher in mosquito positive containers compared to mosquito negative containers 

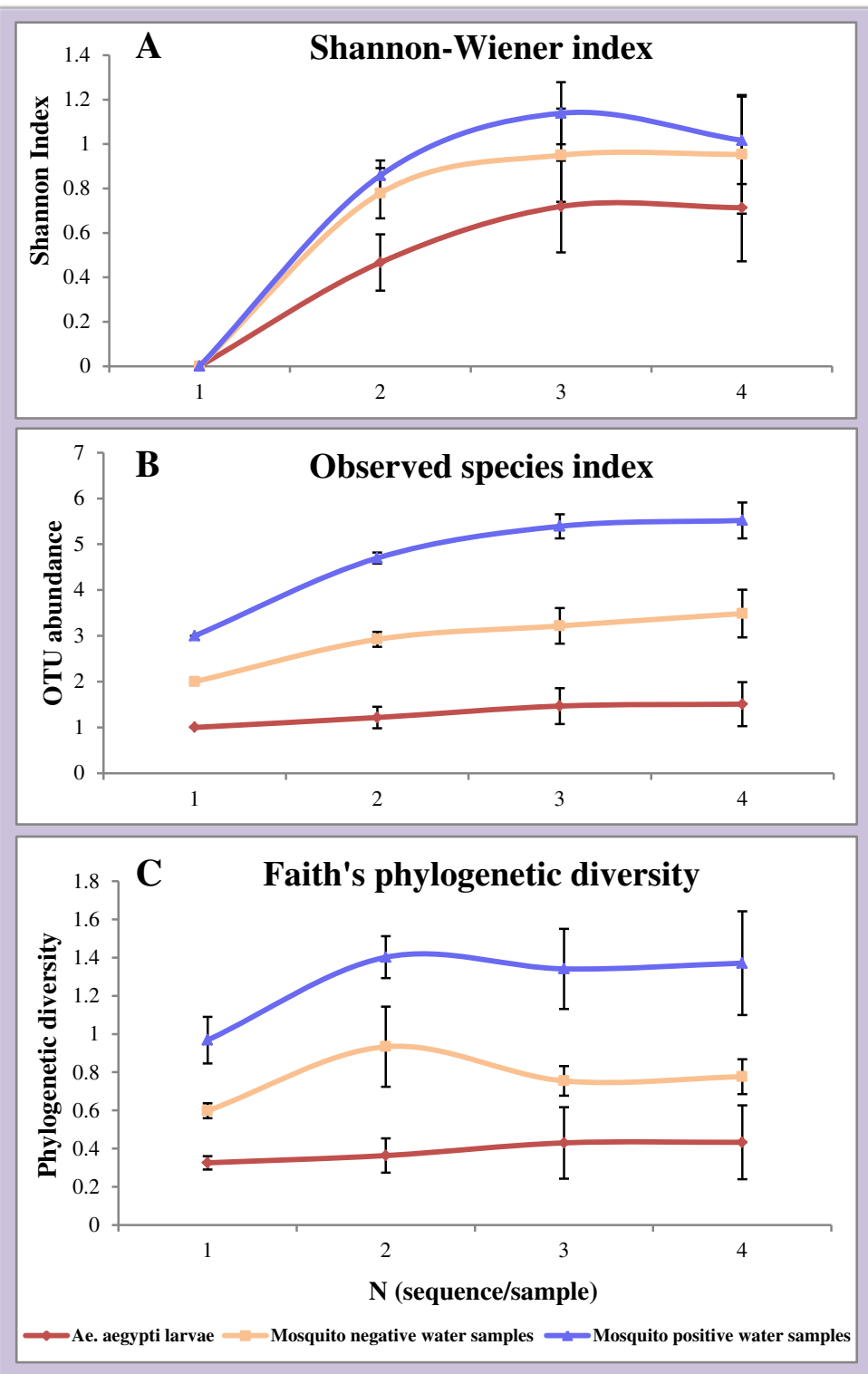

Figure 1 Alpha diversity rarefaction curves of bacterial OTUs from TTGE. Alpha diversity rarefaction curves of bacterial OTUs from Ae. aegypti larvae, mosquito positive and mosquito negative water samples analyzed by TTGE. This is based on Shannon-Wiener index (A), Observed species index (B) and Faith's phylogenetic diversity (C). Curves represent mean diversity indices for each sample, and error bars represent standard error of means.

across all three indices, but the difference was not statistically significant $(\mathrm{p}>0.05)$.

\section{Beta diversity}

The unweighted UniFrac distance metrics was used to compare OTU diversity between samples. The TTGE results showed significantly different and lower bacterial diversity in Ae. aegypti larvae compared to mosquito positive $(\mathrm{p}<0.0001)$ and negative $(\mathrm{p}<0.0001)$ water samples (Figure 2). There was no significant difference in bacterial diversity between mosquito positive and negative water samples $(\mathrm{p}>0.05)$. The PCoA captured over $44 \%$ of variation in UniFrac distances, and revealed distinct clustering patterns of Ae. aegypti larvae and mosquito positive water samples (Figure 3). The PCoA plot shows a distinct cluster of Ae. aegypti larvae towards the right of the first plot between PCo1 and PCo2, as well as the second plot between PCo1 and PCo3. This cluster is also visible towards the left of the third plot between PCo2 and PCo3 (Figure 3). On all three PCoA plots, mosquito positive water samples cluster towards the bottom left, separately from mosquito larvae (Figure 3). The mosquito negative water samples seem to spread out over all three plots without any distinct clustering (Figure 3 ). The adonis test, 


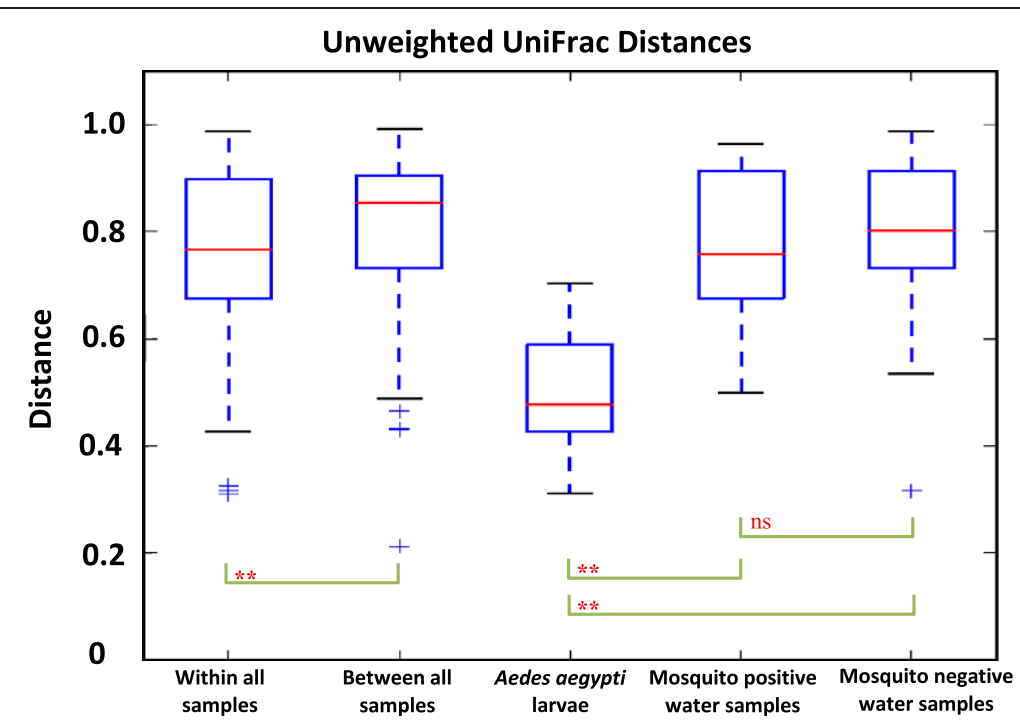

Figure 2 Boxplots of unweighted UniFrac distances of bacterial OTUs from TTGE. Boxplots show distribution of unweighted Unifrac distances of bacterial operational taxonomic units (OTUs) within and between Ae. aegypti larvae, mosquito positive and mosquito negative water samples. Brackets show outcomes of two-sample t-test comparisons of unweighted UniFrac distances; ${ }^{* * P}<0.01$; ns, not significant.

used to test the strength and significance of sample clustering, showed that $20 \%$ of the variation explained by PCoA was statistically significant $\left(\mathrm{R}^{2}=0.20, \mathrm{p}<0.001\right)$. This indicates that the largest amount of variation, explained by PCo1 (Figure 3), is statistically significant.

Comparing sequences from cultured water samples, there was also no significant difference in OTU diversity between mosquito positive and mosquito negative samples $(\mathrm{p}>0.05)$.

\section{Bacterial taxa associated with TTGE sequences}

Four bacterial phyla and one candidate phylum (TM7) - a major lineage of bacteria for which no cultured representatives have been found, but whose existence is known from environmental 16S rRNA sequences - were identified from Ae. aegypti larvae and water from domestic water containers (Figure 4). Only two of these phyla, Actinobacteria and Firmicutes, were found across all samples. The most abundant phylum identified from Ae. aegypti samples, was Firmicutes (52\%), followed by Actinobacteria (25\%). Other bacteria phyla (not broken down in QIIME) made up 18\% of the mosquito samples and the remaining $5 \%$ were unclassified sequences. Proteobacteria was the predominant phylum in both mosquito positive and negative water samples. It constituted $50 \%$ of bacteria phyla found in mosquito positive water samples, followed by Actinobacteria (13\%), Bacteroidetes (9\%), Firmicutes (2\%), TM7 (2\%), other phyla (20\%), and unclassified sequences (4\%). In the mosquito negative water samples, Proteobacteria made up $33 \%$ of the total bacteria phyla, followed by Actinobacteria (18\%), Bacteroidetes (18\%), Firmicutes (2\%), TM7 (1\%), other phyla $(21 \%)$ and unclassified sequences $(7 \%)$.
Figure 5 shows the number of bacterial taxa obtained from TTGE sequences. A total of 24 taxa were identified overall, seven were identified in larval samples, 16 in mosquito positive and 13 in mosquito negative water samples (Figures 4 and 5). Eight of these taxa were shared between mosquito positive and negative water samples (Figure 5), while only two taxa, unclassified Bacilli and Actinomycetales, were common to Ae. aegypti larvae and both mosquito positive and negative water samples (Figures 4 and 5). Other than the two bacterial taxa common to all three samples, no other taxon was shared between Ae. aegypti larvae and water from either mosquito positive or negative containers (Figure 5). Unclassified Bacilli (26\%) were the most abundant bacterial taxa in Ae. aegypti larvae, closely followed by unclassified Actinomycetales (25\%) and Clostridium (11\%). The remaining 38\% comprised unclassified Clostridiales (8\%), Brevibacillus (4\%), unclassified Bacillaceae (1\%), unclassified Firmicutes (1\%), other bacterial genera (19\%) and unclassified sequences (5\%). Unclassified Comamonadaceae (15\%) were the most abundant in mosquito positive water samples, followed by Acinetobacter (13\%), unclassified Proteobacteria (13\%), unclassified Microbacteraceae (9\%), Flavobacterium (9\%), and unclassified Actinomycetales (4\%). The remaining 37\% comprised other bacterial genera (20\%), unclassified sequences (4\%) and other small bacterial taxa contributing $1-2 \%$ of total bacteria genera (Figure 4). Similar groups of bacteria dominated mosquito negative water samples, with Flavobacterium (17\%) being the most dominant, followed by unclassified Comamonadaceae (10\%), unclassified Microbacteraceae (10\%), unclassified Actinomycetales (8\%), unclassified Sphingomonadales (8\%), unclassified 

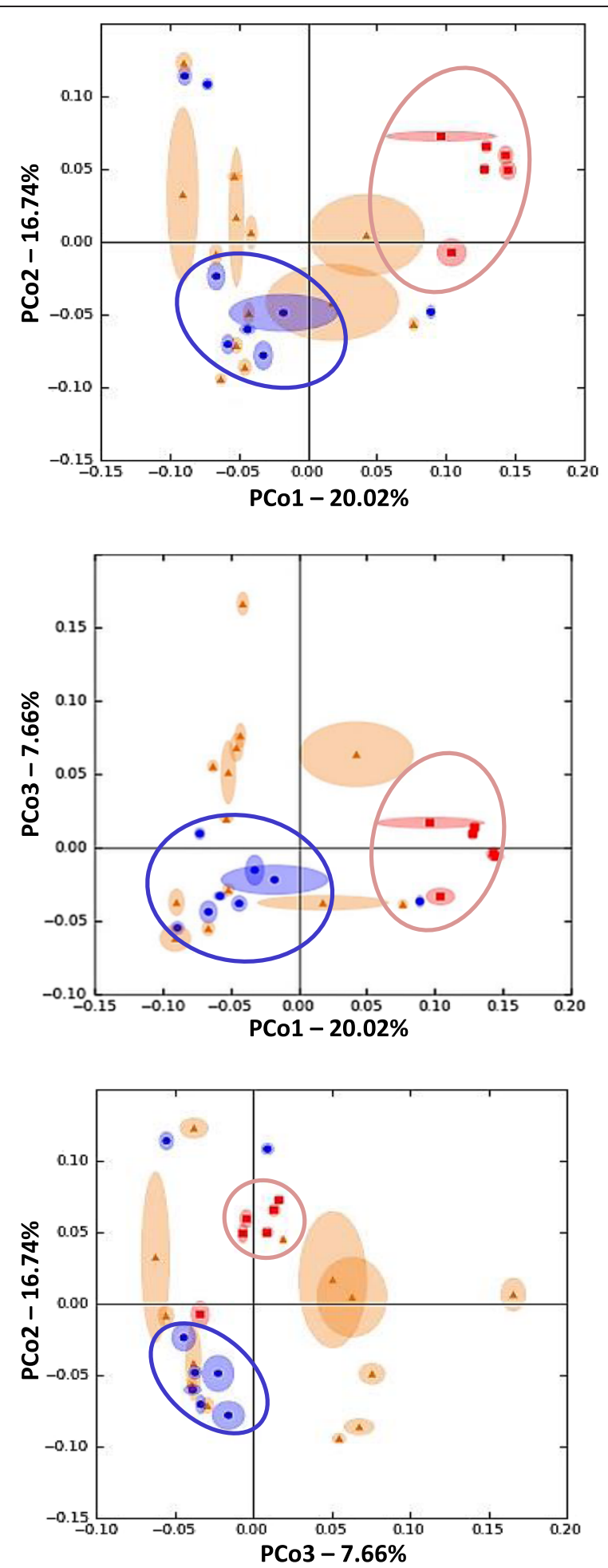

$\Delta$ Mosquito negative water samples

- Mosquito positive water samples a aegypti larvae

Figure 3 PCoA of unweighted UniFrac distances between samples analyzed by TTGE. Principal coordinate analysis of unweighted UniFrac distances between Ae. aegypti larvae and water from mosquito positive and negative containers. Distinct clusters of larval samples (red) and mosquito positive water samples (blue) are captured on all three PCoA plots. Mosquito negative water samples (orange) do not show any distinct clusters on any of the plots. Each axis show percentage of variation explained. Each data point consists of a central point surrounded by ellipsoids that indicate variation in UniFrac distances from rarefaction. This demonstrates that the clustering pattern holds up to subsampling. 


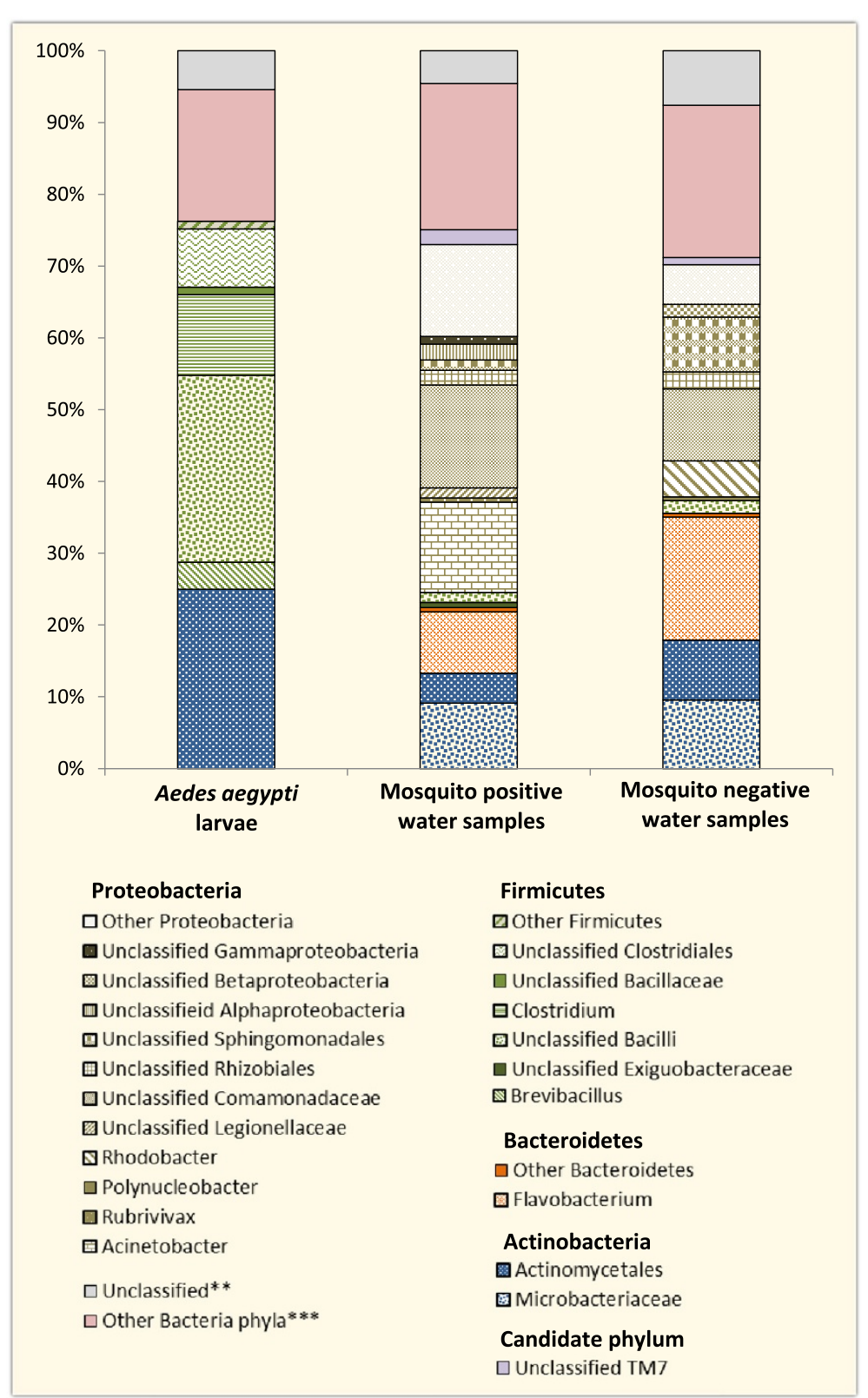

Figure 4 Relative abundance of bacterial taxa obtained from TTGE sequences. Bars show mean relative abundance of the different bacterial taxa isolated by TTGE from Ae. aegypti larvae, mosquito positive and mosquito negative water samples. **Sequences that were not classified by Qiime. ***Low abundance phyla that are automatically grouped together by QIIME.

Proteobacteria (6\%), and Rhodobacter (5\%). The remaining $36 \%$ comprised other bacterial taxa (21\%), unclassified sequences (7\%), and small bacterial taxa contributing $1-2 \%$ of total bacterial taxa (Figure 4).

\section{Bacterial taxa associated with sequences from cultured water samples}

Thirteen bacterial taxa belonging to the phylum Proteobacteria were identified from both mosquito positive and negative water samples (Figure 6). Bacteria belonging to the class Gammaproteobacteria made up $85 \%(n=11)$ of the identified taxa. The other 15\% ( $\mathrm{n}=2)$ comprised members of the class Betaproteobacteria and were only isolated from mosquito positive water samples (Figure 6). Four of the identified bacterial taxa were exclusively from mosquito positive water samples, three from mosquito negative water samples, and six were common to both. Acinetobacter was the most common bacterial genera isolated from mosquito positive water samples. It constituted $37 \%$ of the identified bacterial taxa, followed by Pseudomonas (20\%), Comamonas (13\%), and unclassified Enterobacteriaceae (12\%). The remaining 18\% comprised small bacterial taxa, 


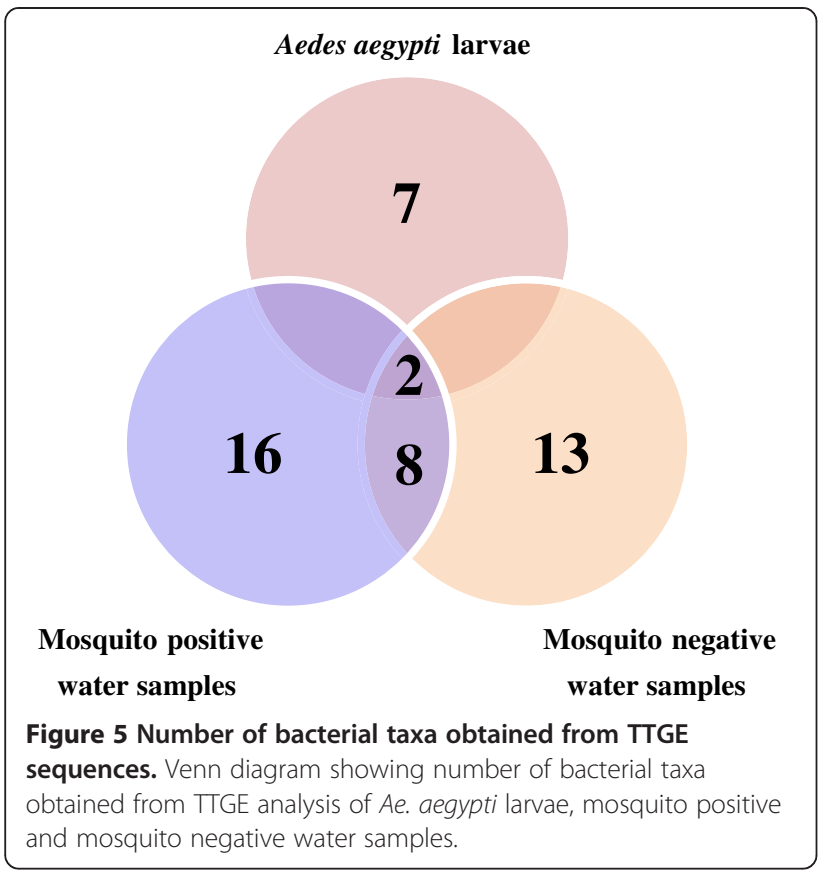

including Escherichia/Shigella, making up between 0.8 $5 \%$ of identified bacterial taxa (Figure 6). Acinetobacter also dominated mosquito negative water samples, making up $27 \%$ of identified bacterial taxa. This was followed by unclassified Enterobacteriaceae (25\%), Stenotrophomonas (20\%) and Escherichia/Shigella (9\%). The remaining $19 \%$ was made up of unclassified sequences (7\%), and small bacterial genera contributing $1-4 \%$ of identified bacterial taxa.

\section{Discussion}

Very sparse information is available on the nature of the microbial community associated with Ae. aegypti larvae in domestic water containers in and around human dwellings. Following our previous study [19], we hypothesized that the bacteria in Ae. aegypti infested containers are determinants of Ae. aegypti production and thus may constitute a major proportion of the larval microbiota. To test this, we utilized the $16 \mathrm{~S}$ rRNA-TTGE to comparatively assess the bacterial communities associated with Ae. aegypti larvae and water from these containers. In addition to TTGE, water samples were cultured for enteric bacteria. This, to the best of our knowledge, is the first study to compare bacterial communities associated with $A e$. aegypti larvae and water from domestic containers.

Aedes aegypti larvae showed significantly lower OTU abundance (Figures 1 and 2) compared to water samples from domestic containers. This low abundance of bacterial taxa in mosquitoes compared to their breeding habitats has been frequently reported $[5,31,32]$, indicating that mosquito larvae naturally have a low number of bacterial taxa. It may also mean that the majority of the bacterial

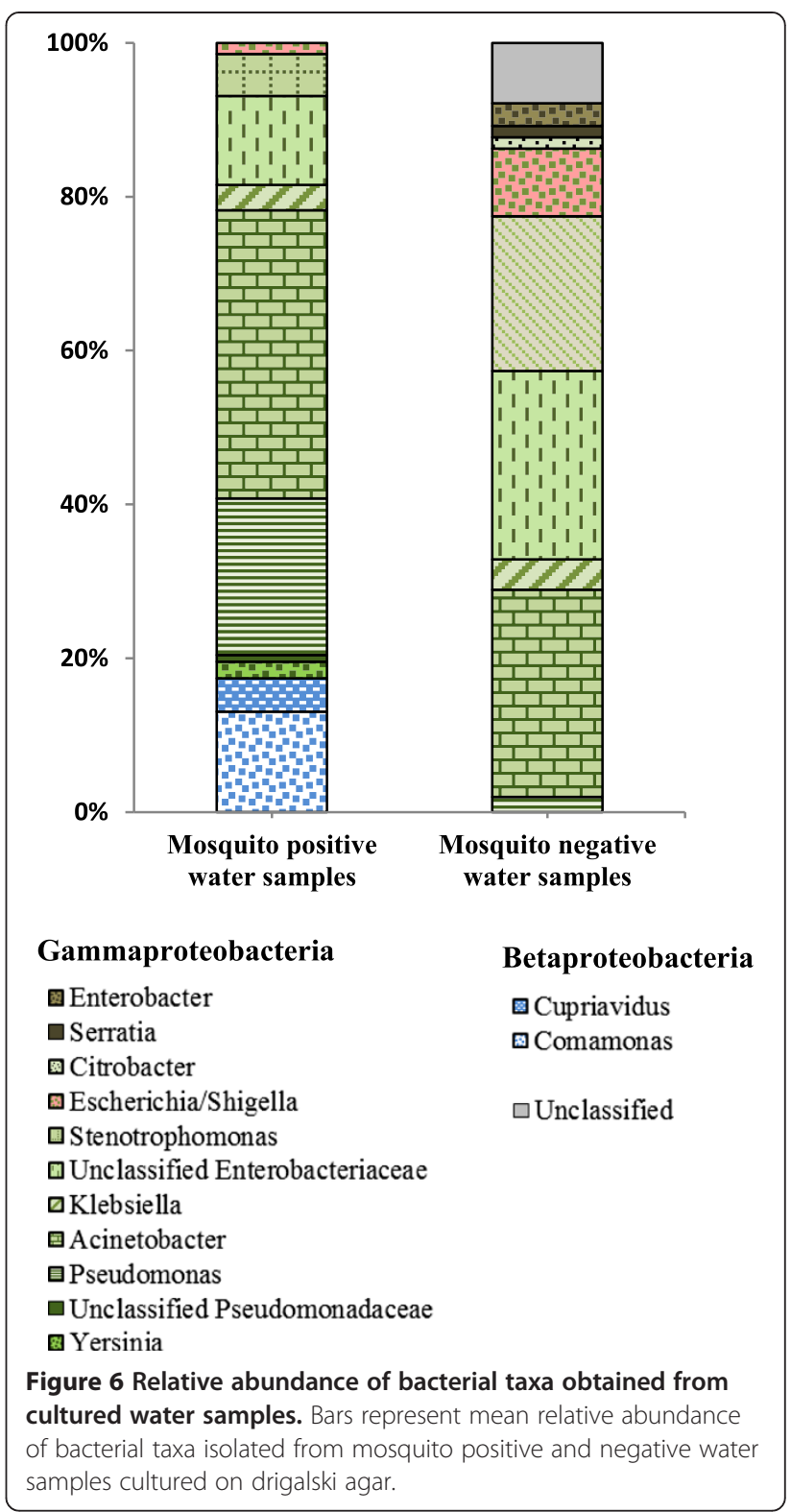

taxa within mosquito larvae are yet to be described, or that methods used for screening are not sufficient to obtain the whole picture of larval microbiota. While larvae consume bacteria, other microbes such as algae and fungi may contribute more significantly to their diet [33,34], which could be another reason for low bacterial OTU abundance.

Although the impact of mosquito larvae on bacterial abundance and diversity in laboratory and field microcosms have been variable [35], most studies report that the presence of larvae affects the bacterial diversity in breeding habitats [36-38]. Our study showed significantly different bacterial diversity between larvae and water samples (Figures 2, 3, and 4), with little overlap between the bacterial communities. This may be because bacteria already present in the larvae dominate and control the 
larval microbiota, thereby inhibiting establishment of new bacteria. Factors such as developmental stage, tissue tropism, genetics, dynamics of intra- and inter-specific interactions, as well as environmental factors are thought to influence the bacterial diversity within mosquitoes [10]. In humans and other mammals, gut microbiota play an important role in 'colony resistance', where they prevent colonization by other bacteria or pathogens [9]. There is some evidence of this 'colony resistance' in insects $[9,10]$, as mosquito gut bacteria can either support or suppress the growth of other species by producing inhibitory factors [39]. Bacteria free in the mosquito gut lumen might evoke a host immune-defense response, or modify the gut environment to inhibit development of other bacteria $[10,40]$. It is also possible that bacteria taken up by late larval instars from their breeding containers are unable to colonize the mosquitoes at this stage of larval development. This is possible due to the selective, competitive or protective mechanisms elicited by established bacteria that may have been acquired at early stages of development. Internal competition among bacterial species could also be an explanation, as predominant bacterial taxa in mosquitoes may have some competitive advantage over other taxa [41].

Overall, four bacterial phyla and one candidate phylum (TM7) were identified from Ae. aegypti larvae and water samples (Figure 4). Only two of these phyla, Firmicutes and Actinobacteria, were associated with both Ae. aegypti larvae and water from their breeding containers, suggesting possible dominance and control of larval bacterial flora by these two phyla. The majority of the bacteria isolated from Ae. aegypti larvae belonged to the classes Bacilli (Bacillaceae, Brevibacillus \& unclassified bacilli), Actinobacteria (Microbacteriaceae \& unclassified Actinomycetales) and Clostridia (Clostridium \& unclassified Clostridiales), with Bacilli being the most predominant. This is consistent with findings from other studies where bacteria belonging to these classes have been identified in different mosquito species [15,42-45] including Ae. aegypti [41,46-50]. One study on the midgut microbiota of Ae. aegypti larvae collected from natural breeding habitats in Thailand identified Bacilli (Bacillus cereus) as the most predominant bacterial class [48]. In contrast, Gammaproteobacteria was the most abundant class (64\%) in Ae. aegypti larvae collected from domestic containers in Pune and Ahemedabad, India [9]. The TTGE results in this study did not identify bacteria from this class or phylum in Ae. aegypti larvae. This may be due to differences in location or techniques used for isolating bacteria, or both.

Proteobacteria was the most predominant phylum in both mosquito positive and negative water samples. The TTGE results showed that Gammaproteobacteria (Acinetobacter, unclassified Gammaproteobacteria, and unclassified Legionellaceae), Betaproteobacteria
(Comamonadaceae, Polynucleobacter, Rubirivivax, and unclassified betaproteobacteria) and Actinobacteria (Actinomycetales, and Microbacteriaceae), were the most abundant bacterial classes in mosquito positive water samples, with Gammaproteobacteria dominating. Flavobacteria (Flavobacterium), Alphaproteobacteria (Rhodobacter, unclassified Sphingomonadales, and unclassified Rhizobiales), and Betaproteobacteria (unclassified Comaonadaceae, unclassified Betaproteobacteri and Polynucleobacter) were the most abundant in mosquito negative water samples, with Flavobacteria dominating. Enteric bacteria belonging to the class Gammaproteobacteria were isolated from both mosquito positive and negative water samples by selective bacterial culture (Figure 6). This class constituted 15\% of the total bacterial population isolated from mosquito positive water samples using TTGE, but was not identified in mosquito negative samples (Figure 4). It may have constituted the 'other bacterial phyla' not broken down in QIIME due to negligible proportions. This indicates that the concentration of Gammaproteobacteria may have been below TTGE detection limit, which is still debatable [18], or outweighed by high concentrations of other competing bacterial DNA [51].

Some bacteria are known to pass through $0.45 \mu \mathrm{m}$ pore size membrane filters impacting bacterial density and, to a lesser extent, diversity. Hence some bacteria in this study may have been lost during filtration. Nonetheless, our results are in line with those of similar studies conducted previously. Studies on the bacterial composition of water samples from domestic containers have understandably focused on fecal contamination [19,52-54], providing little information on general bacterial composition. In the one study conducted so far, majority of the bacteria isolated from Ae. aegypti infested domestic containers were Proteobacteria, predominantly Gammaproteobacteria [9] which is in line with results reported here. In other mosquito habitats such as tree holes, tyres, discarded containers, plant pots, and laboratory mesocosms, Proteobacteria have also been shown to be predominant $[5,9,44]$. Isolation of enteric bacteria from Ae. aegypti infested domestic water containers supports findings from our earlier study [19] where these containers were more likely to be contaminated with $E$. coli (an enteric bacteria) than not. This suggests that enteric bacteria may play a role in Ae. aegypti infestation in this setting.

\section{Conclusions}

We present for the first time results of the bacterial composition of fourth-instar Ae. aegypti larvae and water from Ae. aegypti infested domestic water containers. Aedes aegypti had significantly lower OTU abundance compared to mosquito positive water samples. There was no significant difference in OTU abundance between larvae and 
mosquito negative water samples or between mosquito positive and negative water samples. Larval samples had significantly different OTU diversity compared to mosquito positive and negative water samples, with no significant difference between mosquito positive and negative water samples. The TTGE analysis identified a total of 24 bacterial taxa belonging to the phyla Proteobacteria, Firmicutes, Actinobacteria, Bacteroidetes and TM7 (candidate phylum). Seven of these taxa were identified in $A e$. aegypti larvae, 16 in mosquito positive and 13 in mosquito negative water samples. Eight of the bacterial taxa were common to both mosquito positive and negative water samples, while only two, Firmicutes and Actinobacteria, were common to both larvae and water samples. The lower bacterial abundance in late instar larvae compared to water samples could be due to dominance and control of larval flora by bacteria that may have been established during earlier stages of development. Further investigation of the mosquito-bacteria interactions at all larval stages is needed to understand the dynamics involved. Enteric bacteria were sparsely represented by TTGE, but were isolated from both mosquito positive and negative water samples by selective culture. Isolation of enteric bacteria from water samples in this study supports our previous results of E. coli contamination in Ae. aegypti infested domestic containers in this setting. Studies are needed to understand the role of enteric bacteria in Ae aegypti infestation of these containers. We also show that TTGE alone may not sufficiently describe the bacterial communities in mosquito and water samples. Supplementing with culture-dependent methods presents a better picture of the bacterial communities.

\section{Abbreviations \\ DA: Drigalski agar; DGGE: Denaturing gradient gel electrophoresis; OTU: Operational taxonomic units; PCOA: Principal coordinates analysis; PyNAST: Python nearest alignment space termination; QIIME: Quantitative insights into microbial ecology; RDP: Ribosomal database project; SSU: Small subunit; Tris-EDTA (TE): Ethylenediaminetetraacetic acid-Tris(hydroxymethyl) aminomethane; TSA: Trypticase soy agar; TSB: Tryptic soy broth; TTGE: Temporal temperature gradient gel electrophoresis: UPGMA: Unweighted pair group method with arithmetic mean.}

\section{Competing interests}

The authors declare that they have no competing interests.

\section{Authors' contributions}

ND, HJO, and TAS conceived and designed the study. ND conducted the field and laboratory work, analyzed the samples and data, and drafted the manuscript. EJB developed the protocol for processing samples and supervised sample analyses. SM supervised sample analyses. All authors reviewed and approved the final manuscript.

\section{Acknowledgements}

This study was made possible with funding from the Research Council of Norway (RCN). We thank our collaborating institutions: The Provincial health office, Khon Kaen, District health office, Manchakhiri, and the mosquito lab, Department of Entomology, Kasetsart University, Bangkok for help with facilitating the study. We are grateful to Institut de Recherche pour le Développement (IRD), University Montpellier 1 and Laboratoire d'Hygiène hospitalière, Centre Hospitalier Régional Universitaire de Montpellier,
Montpellier, France for letting us process our samples in their labs. We thank all the Village health volunteers from Waileum village, Manchakhiri district, Khon Kaen for their assistance during sample collection. We thank Nanthasane Vannavong for help with logistics during the field collections and Sara Romano-Bertrand for assistance and technical advice during the laboratory work.

\section{Author details}

${ }^{1}$ Department of Mathematical Sciences and Technology, Norwegian University of Life Sciences, Ås, Norway. 'Laboratoire d'Hygiène hospitalière, Centre Hospitalier Régional Universitaire de Montpellier, Montpellier, France. ${ }^{3}$ UMR5119, Université Montpellier 1, Montpellier, France. ${ }^{4}$ IRD2 Institut de Recherche pour le Développement (IRD), UMR-MD3, Université Montpellier 1, Montpellier, France. ${ }^{5}$ SARChl Chair, Institute for Water and Waste Water Technology, Durban University of Technology, Durban, South Africa. ${ }^{6}$ Institut de Recherche pour le Développement (IRD), Maladies Infectieuses et Vecteurs, Ecologie, Génétique, Evolution et Contrôle (IRD 224-CNRS 5290 UM1-UM2), Montpellier Cedex 5, France. ${ }^{7}$ Department of Entomology, Faculty of Agriculture, Kasetsart University, Bangkok 10900, Thailand.

Received: 14 May 2014 Accepted: 19 August 2014

Published: 24 August 2014

\section{References}

1. Simmons CP, Farrar JJ, Nguyen V, Wills B: Dengue. N Engl J Med 2012, 366 (15):1423-1432.

2. Chareonviriyaphap T, Akratanakul P, Nettanomsak S, Huntamai S: Larval habitats and distribution patterns of Aedes aegypti (Linnaeus) and Aedes albopictus (Skuse), in Thailand. Southeast Asian J Trop Med Public Health 2003, 34(3):529-535.

3. Aziz AT, Dieng H, Ahmad AH, Mahyoub JA, Turkistani AM, Mesed H, Koshike S, Satho T, Salmah MRC, Ahmad H, Zuharah WF, Ramli AS, Miake F: Household survey of container-breeding mosquitoes and climatic factors influencing the prevalence of Aedes aegypti (Diptera: Culicidae) in Makkah City, Saudi Arabia. Asian Pac J Trop Biomed 2012, 2(11):849-857.

4. Hiscox A, Kaye A, Vongphayloth K, Banks I, Piffer M, Khammanithong P, Sananikhom P, Kaul S, Hill N, Lindsay SW, Brey PT: Risk factors for the presence of Aedes aegypti and Aedes albopictus in domestic waterholding containers in areas impacted by the Nam Theun 2 hydroelectric project. Laos Am J Trop Med Hyg 2013, 88(6):1070-1078.

5. Ponnusamy L, Xu N, Stav G, Wesson D, Schal C, Apperson C: Diversity of bacterial communities in container habitats of mosquitoes. Microb Ecol 2008, 56(4):593-603.

6. Yee DA, Allgood D, Kneitel JM, Kuehn KA: Constitutive differences between natural and artificial container mosquito habitats: vector communities, resources, microorganisms, and habitat parameters. J Med Entomol 2012, 49(3):482-491.

7. Walker ED, Lawson DL, Merritt RW, Morgan WT, Klug MJ: Nutrient dynamics, bacterial populations, and mosquito productivity in tree hole ecosystems and microcosms. Ecology 1991, 72(5):1529-1546.

8. Walker ED, O'Meara GF, Morgan WT: Bacterial abundance in larval habitats of Aedes albopictus (Diptera: Culicidae) in a Florida cemetery. J Vector Ecol 1996, 21(2):173-177.

9. Apte-Deshpande A, Paingankar M, Gokhale MD, Deobagkar DN: Serratia odorifera a midgut inhabitant of Aedes aegypti mosquito enhances its susceptibility to dengue-2 virus. PLoS One 2012, 7(7):e40401.

10. Minard G, Mavingui $P$, Moro C: Diversity and function of bacterial microbiota in the mosquito holobiont. Parasit Vectors 2013, 6(1):146.

11. Dillon RJ, Dillon VM: The gut bacteria of insects: nonpathogenic interactions. Annu Rev Entomol 2004, 49:71-92.

12. Ricci I, Damiani C, Capone A, DeFreece C, Rossi P, Favia G: Mosquito/ microbiota interactions: from complex relationships to biotechnological perspectives. Curr Opin Microbiol 2012, 15(3):278-284.

13. Dong Y, Manfredini F, Dimopoulos G: Implication of the mosquito midgut microbiota in the defense against malaria parasites. PLOS Pathog 2009, 5(5):e1000423

14. Ricci I, Valzano M, Ulissi U, Epis S, Cappelli A, Favia G: Symbiotic control of mosquito borne disease. Pathog Glob Health 2012, 106(7):380-385.

15. Pidiyar VJ, Jangid K, Patole MS, Shouche YS: Studies on cultured and uncultured microbiota of wild Culex quinquefasciatus mosquito midgut 
based on 16s ribosomal RNA gene analysis. Am J Trop Med Hyg 2004, 70(6):597-603.

16. Dharne M, Patole M, Shouche YS: Microbiology of the insect gut:tales from mosquitoes and bees. J Biosci 2006, 31(3):293-295.

17. Lindh JM, Borg-Karlson AK, Faye I: Transstadial and horizontal transfer of bacteria within a colony of Anopheles gambiae (Diptera: Culicidae) and oviposition response to bacteria-containing water. Acta Trop 2008, 107(3):242-250.

18. Romano-Bertrand S, Parer S, Lotthé A, Colson P, Albat B, Jumas-Bilak E: Temporal Temperature Gel Electrophoresis to Survey Pathogenic Bacterial Communities: The Case of Surgical Site Infections. In Gel Electrophoresis Advanced Techniques. Edited by Sameh M. Rijeka, Croatia: InTech open access Publisher; 2012:291-312.

19. Dada N, Vannavong N, Seidu R, Lenhart A, Stenstrom TA, Chareonviriyaphap T, Overgaard HJ: Relationship between Aedes aegypti production and occurrence of Escherichia coli in domestic water storage containers in rural and sub-urban villages in Thailand and Laos. Acta Trop 2013, 126(3):177-185.

20. Rattanarithikul R, Harbach RE, Harrison BA, Panthusiri P, Coleman RE, Richardson JH: Illustrated keys to the mosquitoes of Thailand. VI. Tribe Aedini. Southeast Asian J Trop Med Public Health 2010, 41(Suppl 1):1-225.

21. Manguin S, Ngo CT, Tainchum K, Juntarajumnong W, Chareonviriyaphap T, Michon A-L, Jumas-Bilak E: Bacterial Biodiversity in Midguts of Anopheles Mosquitoes, Malaria Vectors in Southeast Asia. In Anopheles mosquitoesNew insights into malaria vectors. Edited by Manguin S. Rijeka, Croatia: InTech open access Publisher; 2013:549-576.

22. Caporaso JG, Kuczynski J, Stombaugh J, Bittinger K, Bushman FD, Costello EK, Fierer N, Pena AG, Goodrich JK, Gordon Jl, Huttley GA, Kelley ST, Knights D, Koenig JE, Ley RE, Lozupone CA, McDonald D, Muegge BD, Pirrung M, Reeder J, Sevinsky JR, Turnbaugh PJ, Walters WA, Widmann J, Yatsunenko T, Zaneveld J, Knight R: QIIME allows analysis of high-throughput community sequencing data. Nat Methods 2010, 7(5):335-336.

23. Edgar RC: Search and clustering orders of magnitude faster than BLAST. Bioinformatics 2010, 26(19):2460-2461.

24. Wang Q, Garrity GM, Tiedje JM, Cole JR: Naive Bayesian classifier for rapid assignment of rRNA sequences into the new bacterial taxonomy. Appl Environ Microbiol 2007, 73(16):5261-5267.

25. McDonald D, Price MN, Goodrich J, Nawrocki EP, DeSantis TZ, Probst A, Andersen GL, Knight R, Hugenholtz P: An improved Greengenes taxonomy with explicit ranks for ecological and evolutionary analyses of bacteria and archaea. ISME J 2012, 6(3):610-618.

26. DeSantis TZ, Hugenholtz P, Larsen N, Rojas M, Brodie EL, Keller K, Huber T, Dalevi D, Hu P, Andersen GL: Greengenes, a chimera-checked 16S rRNA gene database and workbench compatible with ARB. Appl Environ Microbiol 2006, 72(7):5069-5072.

27. Caporaso JG, Bittinger K, Bushman FD, DeSantis TZ, Andersen GL, Knight R: PyNAST: a flexible tool for aligning sequences to a template alignment. Bioinformatics 2010, 26(2):266-267.

28. Price MN, Dehal PS, Arkin AP: FastTree 2 - approximately maximum-likelihood trees for large alignments. PLoS One 2010, 5(3):e9490.

29. Faith DP: Conservation evaluation and phylogenetic diversity. Biol Conserv 1992, 61(1):1-10

30. Lozupone C, Knight R: UniFrac: a new phylogenetic method for comparing microbial communities. Appl Environ Microbiol 2005, 71(12):8228-8235.

31. Coon KL, Vogel KJ, Brown MR, Strand MR: Mosquitoes rely on their gut microbiota for development. Mol Ecol 2014, 23(11):2727-2739.

32. Osei-Poku J, Mbogo CM, Palmer WJ, Jiggins FM: Deep sequencing reveals extensive variation in the gut microbiota of wild mosquitoes from Kenya. Mol Ecol 2012, 21(20):5138-5150

33. Gimnig JE, Ombok M, Otieno S, Kaufman MG, Vulule JM, Walker ED: Density-dependent development of Anopheles gambiae (Diptera: Culicidae) larvae in artificial habitats. J Med Entomol 2002, 39(1):162-172.

34. Kaufman MG, Wanja E, Maknojia S, Bayoh MN, Vulule JM, Walker ED: Importance of algal biomass to growth and development of Anopheles gambiae larvae. J Med Entomol 2006, 43(4):669-676.

35. Kaufman MG, Walker ED, Smith TW, Merritt RW, Klug MJ: Effects of larval mosquitoes (Aedes triseriatus) and stemflow on microbial community dynamics in container habitats. Appl Environ Microbiol 1999, 65(6):2661-2673.

36. Zouache K, Raharimalala FN, Raquin V, Tran-Van V, Raveloson LHR, Ravelonandro P, Mavingui P: Bacterial diversity of field-caught mosquitoes, Aedes albopictus and Aedes aegypti, from different geographic regions of Madagascar. FEMS Microbiol Ecol 2011, 75(3):377-389.

37. Boissière A, Tchioffo MT, Bachar D, Abate L, Marie A, Nsango SE, Shahbazkia HR, Awono-Ambene PH, Levashina EA, Christen R, Morlais I: Midgut microbiota of the malaria mosquito vector Anopheles gambiae and interactions with Plasmodium falciparum infection. PLoS Pathog 2012, 8(5):e1002742.

38. Xu Y, Chen S, Kaufman MG, Maknojia S, Bagdasarian M, Walker ED: Bacterial community structure in tree hole habitats of Ochlerotatus triseriatus: influences of larval feeding. J Am Mosa Control Assoc 2008, 24(2):219-227.

39. Terenius $\mathrm{O}$, Lindh JM, Eriksson-Gonzales K, Bussière L, Laugen AT, Bergquist $\mathrm{H}$, Titanji K, Faye I: Midgut bacterial dynamics in Aedes aegypti. FEMS Microbiol Ecol 2012, 80(3):556-565.

40. Demaio J, Pumpuni CB, Kent M, Beier JC: The midgut bacterial flora of wild Aedes triseriatus, Culex pipiens, and Psorophora columbiae mosquitoes. Am J Trop Med Hyg 1996, 54(2):219-223.

41. Gusmão DS, Santos AV, Marini DC, Bacci M Jr, Berbert-Molina MA, Lemos FJA: Culture-dependent and culture-independent characterization of microorganisms associated with Aedes aegypti (Diptera: Culicidae) (L.) and dynamics of bacterial colonization in the midgut. Acta Trop 2010, 115(3):275-281.

42. Chandel K, Mendki MJ, Parikh RY, Kulkarni G, Tikar SN, Sukumaran D, Prakash S, Parashar BD, Shouche YS, Veer V: Midgut microbial community of Culex quinquefasciatus mosquito populations from India. PLoS One 2013, 8(11):e80453.

43. Valiente Moro C, Tran FH, Nantenaina Raharimalala F, Ravelonandro P, Mavingui P: Diversity of culturable bacteria including Pantoea in wild mosquito Aedes albopictus. BMC Microbiol 2013, 13(1):70.

44. Duguma D, Rugman-Jones P, Kaufman MG, Hall MW, Neufeld JD, Stouthamer R, Walton WE: Bacterial communities associated with Culex mosquito larvae and two emergent aquatic plants of bioremediation importance. PLoS One 2013, 8(8):e72522.

45. Rani A, Sharma A, Rajagopal R, Adak T, Bhatnagar R: Bacterial diversity analysis of larvae and adult midgut microflora using culture-dependent and culture-independent methods in lab-reared and field-collected Anopheles stephensi-an Asian malarial vector. BMC Microbiol 2009, 9(1):96.

46. Gusmao DS, Santos AV, Marini DC, Russo Ede S, Peixoto AM, Bacci Junior M, Berbert-Molina MA, Lemos FJ: First isolation of microorganisms from the gut diverticulum of Aedes aegypti (Diptera: Culicidae): new perspectives for an insect-bacteria association. Mem Inst Oswaldo Cruz 2007, 102(8):919-924.

47. Ramirez JL, Souza-Neto J, Torres Cosme R, Rovira J, Ortiz A, Pascale JM, Dimopoulos G: Reciprocal tripartite interactions between the Aedes aegypti midgut microbiota, innate immune system and dengue virus influences vector competence. PLoS Negl Trop Dis 2012, 6(3):e1561.

48. Luxananil P, Atomi H, Panyim S, Imanaka T: Isolation of bacterial strains colonizable in mosquito larval guts as novel host cells for mosquito control. J Biosci Bioeng 2001, 92(4):342-345.

49. Chouaia B, Rossi P, Montagna M, Ricci I, Crotti E, Damiani C, Epis S, Faye I, Sagnon NF, Alma A, Favia G, Daffonchio D, Bandi C: Molecular evidence for multiple infections as revealed by typing of Asaia bacterial symbionts of four mosquito species. Appl Environ Microbiol 2010, 76(22):7444-7450.

50. Wistreich GA, Chao J: Microorganisms from the Midgut of Larval and Adult Aedes aegypti (Linnaeus). J Insect Pathol 1963, 5:56-60.

51. Ogier JC, Son O, Gruss A, Tailliez P, Delacroix-Buchet A: Identification of the bacterial microflora in dairy products by temporal temperature gradient gel electrophoresis. Appl Environ Microbiol 2002, 68(8):3691-3701.

52. Oswald WE, Lescano AG, Bern C, Calderon MM, Cabrera L, Gilman RH: Fecal contamination of drinking water within Peri-Urban households, Lima. Peru Am J Trop Med Hyg 2007, 77(4):699-704.

53. Han AM, Oo KN, Midorikawa Y, Shwe S: Contamination of drinking water during collection and storage. Trop Geogr Med 1989, 41(2):138-140.

54. Mattioli MC, Boehm AB, Davis J, Harris AR, Mrisho M, Pickering AJ: Enteric pathogens in stored drinking water and on caregiver's hands in Tanzanian households with and without reported cases of child diarrhea. PLoS One 2014, 9(1):e84939.

doi:10.1186/1756-3305-7-391

Cite this article as: Dada et al:: Comparative assessment of the bacterial communities associated with Aedes aegypti larvae and water from domestic water storage containers. Parasites \& Vectors 2014 7:391. 